Revista Médica Sinergia

Vol. 6, Núm. 8, agosto 2021, e698

\title{
Síndrome metabólico en la edad pediátrica: ¿qué sabemos hasta el momento?
}

\author{
Metabolic syndrome in pediatric age: what do we know so far?
}

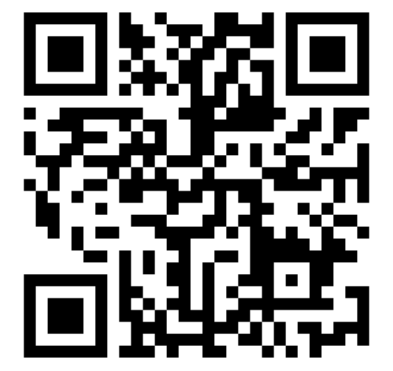

Recibido 22/06/2021
${ }^{1}$ Dra. Stephanie León Quirós Investigadora independiente, Heredia, Costa Rica

(D) https://orcid.org/0000-0002-4464-9501

${ }^{2}$ Dra. Amanda Vargas Guzmán Investigadora independiente, San José, Costa Rica

(D) https://orcid.org/000-0002-2093-1385

${ }^{3}$ Dra. Michelle Fung Fallas Investigadora independiente, San José, Costa Rica

(iD https://orcid.org/0000-0003-0664-6059

Corregido $10 / 07 / 2021$
Aceptado 20/07/2021

\section{RESUMEN}

El síndrome metabólico representa un reto para la salud pública, pues genera un aumento de morbilidad y mortalidad en la población y su prevalencia se encuentra en aumento, en todas las edades. Se debe a una compleja interacción entre factores genéticos, conductuales y ambientales, cuyos efectos nocivos para la salud física del individuo se ha demostrado que inician su afectación desde la vida intrauterina, y se mantienen hasta la edad adulta. Supone un reto diagnóstico en la edad pediátrica, por falta de consenso en su definición y criterios diagnósticos. En cuanto al abordaje también supone un desafío por los múltiples factores a tratar, sin embargo, sí se ha demostrado la importancia de promover en primer lugar la prevención, pues a menor edad y avance de los efectos perjudiciales, mayor es el beneficio que se obtiene, para esto, se entrena a los padres y niños a seguir hábitos saludables de actividad física, patrones de sueño, lactancia materna y posteriormente una dieta saludable. Existen opciones también dirigidas al tratamiento de quien ya lo padece, y una de las estrategias con mejores resultados es el uso de terapia cognitivo-conductual, pero a su vez quedan más objetos de estudio para el futuro.

PALABRAS CLAVE: síndrome metabólico; pediatría; atención primaria de salud; obesidad; prevención primaria. 


\begin{abstract}
The metabolic syndrome represents a challenge for public health, since it generates an increase in morbidity and mortality in the population and its prevalence is increasing at all ages. It is due to a complex interaction between genetic, behavioral and environmental factors whose harmful effects on the physical health of the individual have been shown to start their affectation from intrauterine life and remain until adulthood. It is a diagnostic challenge in pediatric age, due to lack of consensus in its definition and diagnostic criteria. Regarding the approach, it also poses a challenge due to the multiple factors to be treated, however, the importance of promoting prevention in the first place has been demonstrated, since the younger age and the less advance of the harmful effects, the greater benefit obtained. For this, parents and children are trained to follow healthy habits of physical activity, sleep patterns, breastfeeding and subsequently a healthy diet. There are also options aimed at treating those who already suffer from it, and one of the strategies with the best results is the use of cognitive-behavioral therapy, but in turn there are more objects of study for the future.
\end{abstract}

KEYWORDS: metabolic syndrome; pediatric; primary health care; obesity; primary prevention.

\footnotetext{
${ }^{1}$ Médica general, graduada de la Universidad de Costa Rica (UCR). Cód. MED16835. Correo: spleon12@gmail.com

${ }^{2}$ Médica general, graduada de la Universidad de Costa Rica (UCR). Cód. MED16540. Correo: amandavargas93@gmail.com

${ }^{3}$ Médica general, graduada de la Universidad de Costa Rica (UCR). Cód. MED16824. Correo: $\underline{\text { mfungf@gmail.com }}$
}

\section{INTRODUCCIÓN}

Actualmente el sobrepeso y obesidad ocurren con mayor prevalencia y a menor edad, se estima que para el 2030 el $57.8 \%$ de la población mundial tendrá alguno de estos dos padecimientos, incluso, en 2016 se publicó un estudio hecho con población en edad pediátrica en México, en el que la prevalencia de estas condiciones resultó en un $32 \%$. Junto con la resistencia a la insulina, el sobrepeso y la obesidad están catalogados como los mayores predictores de síndrome metabólico.

Este último, también cada vez más prevalente, constituye un importante problema de salud pública, porque aumenta dos veces el riesgo de enfermedad cardiovascular, cinco veces el riesgo de diabetes mellitus 2, las cuales aumentan la morbi - mortalidad de los individuos que las padecen.

Por su parte, la población pediátrica no se encuentra exenta de estas condiciones de salud, e incluso presenta más riesgo de desarrollar estas complicaciones con el tiempo, por lo que un abordaje temprano es vital. El impacto en salud pública en Costa Rica, de acuerdo a un estudio publicado en 2015 en el que se documentó la prevalencia de este síndrome en población adulta en la región centroamericana, Costa Rica ocupa el primer lugar con un $35.1 \%$ de prevalencia de síndrome metabólico (1-5).

Debido a su rol negativo para la salud pública y su creciente prevalencia, el objetivo de esta revisión es reunir en un solo documento una síntesis de la evidencia científica reciente en relación con el síndrome metabólico en la edad pediátrica, se incluyen sus distintas definiciones, factores predisponentes, parámetros diagnósticos y posibles abordajes, enfocado principalmente en la prevención primaria, excluyendo los abordajes específicos de cada alteración metabólica.

\section{MÉTODO}

Se realizó una revisión bibliográfica de artículos científicos originales, excluyendo 
aquellos artículos que se trataran de revisiones bibliográficas, publicados en los últimos 10 años. Mediante la consulta en las bases de datos ScienceDirect, PubMed, Cochrane, entre otras, accesadas a través del Sistema de Bibliotecas, Documentación e Información (SIBDI) de la Universidad de Costa Rica, la Biblioteca Nacional de Salud y Seguridad Social (BINASSS) y SciELO. Para la búsqueda se utilizaron como palabras claves: "síndrome metabólico", "pediatría", "metabolic syndrome" y "pediatric".

\section{GENERALIDADES}

El síndrome metabólico no es una enfermedad en sí, sino que refiere a la coexistencia de varios desórdenes metabólicos en un individuo, estos son de los factores de riesgo más peligrosos para enfermedad cardiovascular, pero que son modificables, se detallan a continuación: obesidad central, triglicéridos altos o colesterol de lipoproteínas de alta densidad (HDL) bajo, hipertensión arterial y alteraciones en el metabolismo e la glucosa. Además del conocido riesgo aumentado de diabetes mellitus y enfermedad cardiovascular que representa este síndrome, también se le ha asociado con aumentar la prevalencia de asma, apnea del sueño, malignidades y mortalidad en general (5-7).

Su prevalencia se encuentra en aumento, y para entenderlo se deben analizar los factores metabólicos que lo componen por separado, iniciando por el aumento notorio de obesidad en la población pediátrica, factores genéticos, estilos de vida sedentarios o con mucho aporte calórico, patrón de sueño, condiciones socioeconómicas e incluso se ha estudiado el papel de la contaminación ambiental $(1,7-$ 9).

\section{FACTORES PREDISPONENTES}

- Hábitos de sueño: malos hábitos de nutrición y sueño tienen estrecha relación con el síndrome metabólico, más allá de un alto aporte calórico debido a una mala alimentación, el metabolismo de lípidos y carbohidratos es regulado por hormonas que se secretan siguiendo el ritmo circadiano. En un estudio realizado con población pediátrica en Grecia se documentó el impacto positivo en la salud de tener buenos hábitos nutricionales y de sueño, sin diferencias significativas entre ellos, pero sí con la salvedad de que tuvieron mayor impacto en aquellos niños que no tenían factores predisponentes para síndrome metabólico, esto se puede interpretar como que es más fácil prevenirlo que tratarlo (8).

Respecto a los hábitos de sueño, se contempla no solo el tiempo dormido, sino también las siestas durante el día y la hora a la que se acuesta en la noche. Dormir pocas horas se relaciona con un aumento de todos los parámetros del síndrome, disponen de más tiempo para comer y tienden a optar por una vida más sedentaria. Además, el sueño corto se traduce, a nivel hormonal, en menos leptina y más grelina, haciendo que el individuo sienta más hambre y tenga preferencia por alimentos altamente calóricos; de igual forma se aumenta la secreción de catecolaminas contribuyendo a un aumento de presión arterial. De acuerdo con un estudio realizado en China, es posible que el resultado de la deprivación de sueño varíe según edad y género. Por otro lado, la hora a la que se duerme por la noche incide en la cantidad de melatonina que se secreta, entre más tarde, menos hormona se libera, esta tiene acción en las células $\beta$ del páncreas, disminuyendo 
la cantidad de insulina durante el sueño, por ende, menos melatonina implica más insulina y eso predispone a diabetes mellitus 2. Por último, tomar siestas durante el día se asocia a dislipidemia e intolerancia a la glucosa, posiblemente porque con la alteración del ciclo circadiano se aumentan los niveles de cortisol $(8,10)$.

- Condiciones socioeconómicas: se ha visto que un bajo nivel socioeconómico se asocia a mala dieta, poca actividad física y a ser más sedentarios, incluso, un mayor nivel socioeconómico en la infancia se asocia a menor riesgo de síndrome metabólico en la adultez, independientemente del nivel socioeconómico que dicha persona posea al alcanzar la edad adulta. De los factores presentes en las familias con menor nivel socioeconómico uno de los que más impacto mostró sobre los niños fue el grado académico de los padres, pues a padres con menor educación se asocia a peores hábitos alimenticios $y$ menos actividades recreativas. De igual manera, se ha estudiado que aquellas familias que, debido a sus condiciones económicas, no tienen seguridad de que van a poder adquirir siempre sus alimentos, optan por opciones de comida de baja calidad nutricional, con alto contenido calórico y azúcar, pero a un menor precio. A su vez, estas familias muestran una tendencia mayor a creer que el llanto del niño siempre es a causa de hambre y crean así un patrón conductual de consuelo alrededor de la comida que podría afectar al infante por el resto de su vida $(11,12)$.

- Contaminación ambiental: esta influye en cada componente del síndrome metabólico de forma individual, de acuerdo a los estudios, el inhalar aire contaminado resulta en estrés oxidativo, inflamación sistémica y desbalance sistema nervioso autonómico, los cuales a su vez se traducen en disfunción endotelial y resistencia a la insulina. No se dispone de tantos estudios en niños, pero se maneja la hipótesis de que son especialmente susceptibles debido a mayor cantidad de actividades al aire libre y a los continuos cambios fisiológicos del desarrollo. Zhang et al. realizaron un estudio con esta población, en China, entre 2013-2015, en el que encontraron que la exposición a la contaminación del aire al largo plazo incide en una mayor prevalencia de síndrome metabólico, con mayor afectación de varones, posiblemente por mayor actividad física al aire libre, sin embargo, hay otras variantes que pueden influir en estos resultados, como el tamaño pulmonar, estilos de vida y factores genéticos, por lo que más estudios se requieren en este campo (7).

- Exposición a diabetes mellitus gestacional (DMG): se ha visto que aquellos hijos de madres que padecieron de esta enfermedad presentan tendencia a una mayor adiposidad corporal total y central, que es la más asociada a aumento de riesgo cardiovascular. Además, en estudios prospectivos se ha documentado la tendencia de estos pacientes a llevar un estilo de vida menos saludable, con poca actividad física y menos consumo de frutas, aun así, si se comparan sus índices de adiposidad contra los de niños no expuestos a la DMG que también tienen mal estilo de vida, resultan mayores los de los infantes expuestos a DMG (13).

- Alimentación en los primeros mil días de vida: según estudios alrededor de la obesidad, se ha documentado que en los primeros mil días se define el patrón de adiposidad del individuo, y que una 
acelerada ganancia de peso para la edad y de peso para la talla se asocian con mayor prevalencia de obesidad en edades futuras, la primera representa la masa grasa y la segunda los depósitos de tejido adiposo. Ante esto se ha estudiado el efecto que tienen la lactancia materna, la fórmula y la ablactación, la evidencia indica que el mantener la lactancia materna y postergar la ablactación hasta al menos los 4 meses de vida tiene menos repercusiones en esa ganancia de peso acelerada. Una de las posibles explicaciones es que el niño que es ablactado muestra una disminución de ingesta de leche materna, a diferencia de los que son alimentados con fórmula que al ablactarlos no se les disminuye la cantidad de fórmula que toman. Además, respecto a la influencia de la ablactación temprana, se ha visto que el impacto que tiene en cuanto a la ganancia de peso varía dependiendo de si antes se alimentaba con lactancia materna o fórmula (14).

- Los estudios muestran resultados variables, pero se ha identificado hasta 6 veces más ganancia de peso en aquellos infantes alimentados con fórmula, sin lactancia materna, y ablactados antes de los 4 meses de edad (14).

\section{DIAGNÓSTICO}

En adultos se debe presentar al menos 3 de los factores de riesgo antes mencionados, sin embargo, no hay claro consenso en cuanto a los valores de los puntos de corte para definirlo en la edad pediátrica, pues no hay suficiente claridad de con qué niveles esos factores metabólicos empiezan a causar un daño en esta población, además que se debe tomar en cuenta que estos pueden variar dependiendo de la etnia y años de vida de cada paciente $(6,9,15)$.
En distintos estudios se ha intentado establecer cuál es la medida antropométrica con mayor valor predictivo para identificar aquellos pacientes con síndrome metabólico en la edad pediátrica, pues hay que tomar en consideración las variantes fisiológicas y anatómicas que se dan durante el desarrollo; se han valorado las diferentes medidas utilizadas en adultos, como la medida de la cintura, circunferencia de cadera, relación cintura-estatura, índice de masa corporal, circunferencia de muñeca, entre otras, y los estudios llegan a distintas conclusiones por lo que aún no se logra un consenso de cuál es la mejor medida a utilizar, sin embargo, parece que, junto a la clásica medida de cintura abdominal, la circunferencia de cadera y la relación cintura estatura pueden ser las de mayor utilidad diagnóstica (15).

Por otra parte, también se han propuesto nuevas opciones, sustentados en diversos estudios que proponen que la contribución de la adiposidad al riesgo metabólico depende de la distribución de la misma, siendo la que se encuentra en la parte superior del cuerpo la más dañina, esto dado que contribuye a una mayor presencia de ácidos grasos libres, los cuales se relacionan directamente con la resistencia a la insulina, un HDL bajo, producción de triglicéridos, estrés oxidativo y disfunción endotelial que conduce a hipertensión arterial; y tomando en cuenta la variabilidad que puede darse en la medida de la cintura, por el estado postprandio, composición anatómica, experiencia del evaluador, entre otras... Formisano, et al. realizaron un estudio con más de quince mil pacientes para identificar si la circunferencia del cuello correlaciona con los factores de riesgo cardiovascular en población pediátrica, ya que esta medida se ha propuesto por tener menos variación que la cintura. Reportan que esta medida es buena identificar pacientes con riesgo metabólico en ambos géneros. En el estudio detallan punto de corte para esta medida, de acuerdo a edad y 
género, varía desde $24.95 \mathrm{~cm}$ en las niñas de 3-4 años, hasta $30.90 \mathrm{~cm}$ en los niños 9-10 años, para las pacientes femeninas este parámetro es menor (9).

Mientras que las algunas de las medidas antropométricas ya se utilizan en cada consulta, los demás parámetros aun no tienen una edad clara en la que se deba iniciar el tamizaje, y depende, en parte, de características en historia clínica y examen físico, que despierten la sospecha en el evaluador, como la presencia de acantosis nigricans o el padecimiento de alguna de las alteraciones metabólicas ya mencionadas, que debe sugerir tamizar por las otras. De acuerdo con la Academia Americana de Pediatría (AAP) se sugiere, con un grado $C$ de evidencia, iniciar el tamizaje de presión arterial a los 3 años, con una medición anual, a excepción de pacientes obesos, diabéticos o con condiciones específicas, como padecimientos o uso de medicamentos que alteran esta medida, en quienes se debe realizar en cada encuentro $(6,15,16)$.

En lo que respecta al perfil lipídico se recomienda tamizar a todos los niños a partir de los 2 años cuando tengan antecedente heredo familiar de enfermedad cardiovascular en menores de 55 años o hipercolesterolemia en los padres, también se sugiere en caso de desconocer antecedentes o si se tiene antecedente personal de los otros factores de riesgo cardiovascular que definen este síndrome, e incluyen también el tabaquismo. De los anteriores el factor de riesgo más sensible y específico es el antecedente de padres con hipercolesterolemia, estos hijos han demostrado una prevalencia de hasta seis veces más que los que tienen padres sin esta condición. Sin embargo, se ha demostrado que de utilizar únicamente un tamizaje dirigido a aquellos que presentan factores de riesgo deja sin diagnóstico muchas dislipidemias moderadas e incluso severas que se podrían haber detectado a tiempo si se utiliza un tamizaje universal, por lo que la Academia Americana de Pediatría en 2017 actualiza sus recomendaciones y sugiere realizar un tamizaje universal entre los 9-11 años y otro entre los 17-21 años $(17,18,19)$.

Uno de los mayores retos para dictaminar el síndrome metabólico, es la falta de consenso en su definición y niveles diagnósticos de cada parámetro. En 2017 un estudio transversal realizado en población mexicana de los 9-13 años, comparó algunos de los puntos de corte propuestos para definir este síndrome en la edad pediátrica, con el fin de identificar cuál de las propuestas detecta una mayor prevalencia de este padecimiento. De acuerdo a sus resultados la definición de Ferranti (tabla 1) es detecta más y la de Weiss (tabla 1) es el que detecta menos, y proponen, basados en que el mayor evento metabólico del síndrome es la resistencia a la insulina, que por sí sola ya representa un riesgo aumentado de desarrollar diabetes mellitus y por ende la importancia de su detección temprana, utilizar un Índice de resistencia a la insulina (HOMA-IR) $\geq 3.4$ para definir aquellos que ya presentaban esta condición. Con lo anterior resultó que $12.4 \%$ de pacientes que no habían sido detectados con los criterios de Ferranti, contra $25.2 \%$ con los de Weiss, además, concluyen que al incluirse esta forma de determinar resistencia a la insulina se disminuyen de forma significativa las discrepancias entre las distintas definiciones (5).

Un estudio realizado con población europea, de 2-9 años, llamado IDEFICS por sus siglas en inglés, propone una evaluación más constante, con dos puntos de corte distintos, uno para identificar aquellos pacientes que se deben monitorear por su alto riesgo y otro para aquellos que ya necesitan intervención, los parámetros son: circunferencia de cintura, presión arterial sistólica o diastólica, triglicéridos o HDL y HOMA-IR a la insulina o glicemia en ayunas; cuando se encuentren superior 0 igual al percentilo 90 se 
encuentran en el grupo de monitoreo y mayor o igual al percentilo 95 en el grupo que amerita acciones, con excepción del HDL que su punto de corte es menor o igual al percentilo 10. Comparan la prevalencia de síndrome metabólico al utilizar los nuevos puntos de corte propuestos contra algunas definiciones ya utilizadas y concluyen que la definición de Cook et al. y Viner et al. (tabla 1) contemplan niveles de glucosa muy elevados por lo que solo unos cuantos cumplen el criterio y por otro lado que la definición de la Federación Internacional de Diabetes (tabla 1) solicita cifras de presión arterial de adultos, razones por las que los puntos de corte propuestos en ese estudio identifican a una mayor cantidad de pacientes (6).

\section{ABORDAJE}

La presencia de factores que predisponen a padecer de síndrome metabólico en la infancia se ha evidenciado que tienden a permanecer a la edad adulta y que su contribución negativa a la salud física y metabólica sí pueden iniciar desde la infancia, contribuyendo a una muerte prematura en la edad adulta, sin embargo, estos pueden ser modificables, por lo que la prevención y abordaje temprano son muy necesarios $(1,8)$.

Los estudios han demostrado que niños expuestos a DMG, a pesar de estar predispuestos a tener una mayor adiposidad, si mantienen hábitos de vida saludables tienen menor adiposidad, incluso pudiendo atenuar la influencia de la exposición a DMG en el útero, lo que demuestra que los factores pueden ser modificables y por ende el impacto que tienen los hábitos de vida (13).

\section{Abordaje preventivo}

No se ha identificado consistentemente cuál es la mejor estrategia de prevención, por ejemplo, McCormick, et al., realizaron un estudio con población latina, de alto riesgo

\begin{tabular}{|l|l|l|l|l|l|l|}
\hline \multicolumn{1}{|c|}{ Tabla 1. Definiciones de síndrome metabólico en la edad pediátrica. } \\
\hline \multicolumn{1}{|c|}{ Parámetros } & Ferranti & Weiss & $\begin{array}{c}\text { Cook et } \\
\text { al. }\end{array}$ & Viner et al. & \multicolumn{1}{|c|}{ FID } & $\begin{array}{c}\text { IDEFICS } \\
\text { (monitoreo) }\end{array}$ \\
\hline $\begin{array}{l}\text { Circunferencia } \\
\text { cintura }\end{array}$ & $\geq \mathrm{P} 75$ & No aplica & $\geq \mathrm{P} 90$ & $\geq \mathrm{P} 95$ & $\geq \mathrm{P} 90$ & $\geq \mathrm{P} 90$ \\
\hline IMC z-score & No aplica & $\geq 2.0$ & No aplica & No aplica & No aplica & No aplica \\
\hline Presión arterial & $\geq \mathrm{P} 90$ & $\geq \mathrm{P} 95$ & $\geq \mathrm{P} 90$ & $\mathrm{PAS} \geq \mathrm{P} 95$ & $\begin{array}{l}\text { PAS } \\
\geq 130 \mathrm{mmHg}\end{array}$ & \\
\hline Colesterol HDL & $\begin{array}{l}\mathrm{PAD} \\
\geq 85 \mathrm{mmHg}\end{array}$ & $\geq \mathrm{P} 90$ & & & & \\
\hline Triglicéridos & $<50 \mathrm{mg} / \mathrm{dl}$ & $\leq \mathrm{P} 5$ & $\leq 40 \mathrm{mg} / \mathrm{dl}$ & $<35 \mathrm{mg} / \mathrm{dl}$ & $<40 \mathrm{mg} / \mathrm{dl}$ & $\leq \mathrm{P} 10$ \\
\hline Colesterol total & $\geq 100 \mathrm{mg} / \mathrm{dl}$ & $\geq \mathrm{P} 95$ & $\geq 110 \mathrm{mg} / \mathrm{dl}$ & $\geq 150 \mathrm{mg} / \mathrm{dl}$ & $\geq 150 \mathrm{mg} / \mathrm{dl}$ & $\geq \mathrm{P} 90$ \\
\hline Glicemia ayunas & No aplica & No aplica & No aplica & $\geq \mathrm{P} 95$ & No aplica & No aplica \\
\hline Hiperinsulinemia & $\geq 110 \mathrm{mg} / \mathrm{dl}$ & $\geq 110 \mathrm{mg} / \mathrm{dl}$ & $\geq 110 \mathrm{mg} / \mathrm{dl}$ & $\geq 110 \mathrm{mg} / \mathrm{dl}$ & $\geq 100 \mathrm{mg} / \mathrm{dl}$ & $\geq \mathrm{P} 90$ \\
\hline HOMA-IR & No aplica & No aplica & No aplica & $\geq 104.2 \mathrm{pmol} / \mathrm{L}$ & No aplica & No aplica \\
\hline
\end{tabular}

Abreviaturas. FID, Federación Internacional de Diabetes; Índice de resistencia a la insulina; IMC, HDL, lipoproteínas de alta densidad; HOMA-IR, índice de masa corporal; PAS, presión arterial sistólica; PAD, presión arterial diastólica.

Fuente. 'Peña-Espinoza BI, Granados-Silvestre M de LÁ, Sánchez-Pozos K, Ortiz-López MG, Menjivar M. Metabolic syndrome in Mexican children: Low effectiveness of diagnostic definitions. Endocrinol Diabetes Nutr (Engl ). 2017;64(7):369-76. ²Ahrens W, Moreno LA, Mårild S, Molnár D, Siani A, De Henauw S, et al. Metabolic syndrome in young children: definitions and results of the IDEFICS study. Int J Obes (Lond). 2014;38 Suppl 2(S2):S4-14. 
para síndrome metabólico, en el que se le dio acompañamiento con visita domiciliar a las madres desde el nacimiento de los niños para brindarles educación sobre una alimentación saludable, sin embargo, este estudio reporta fallo en prevención de sobrepeso y obesidad. Mientras que Reifsnider et al., también con población latina de alto riesgo, realizaron visitas para educación pre y postnatales, pero a diferencia del anterior les educaron no solo en dieta sino en actividad física, hábitos de sueño, salud y desarrollo en general del niño, y tampoco lograron gran impacto en la disminución de prevalencia, al menos en el seguimiento a 1 año que contemplaba el estudio $(20,21)$.

Lo que sí se ha identificado son las conductas que se pueden promover que tiene impacto positivo en la disminución de la prevalencia del síndrome metabólico: promover un estilo de vida activo, con actividad física extracurricular, disminución en el tiempo de uso de pantallas, hábitos de sueño saludables, que contemplen una adecuada duración de sueño según la edad, disminución de siestas durante el día y dormirse antes de las $22: 00$ horas $(8,10,22)$. Respecto a la dieta, se sugiere promover la lactancia materna y, en caso de necesitar fórmula, enseñar a los cuidadores la dosis adecuada para cada niño, así como la importancia de no agregar azúcar. Se ha demostrado asociación entre la lactancia materna y la adecuada ganancia de peso, así como entre el uso de fórmula y el sobrepeso y obesidad, aunque en este punto no todos los estudios concuerdan. Se sugiere una ablactación posterior a los 4 meses de edad, con acompañamiento profesional para la adecuada selección de calidad y cantidad de alimentos. Según Reifsnider et al., en su estudio documentaron que los que presentaban sobrepeso a los 6 meses de edad tuvieron la tendencia a preservar este patrón en el seguimiento hasta el año de edad, a pesar de las intervenciones educativas que realizaron $(14,20,21)$.

Además, un estudio realizado con población italiana se concluye que aquellos que se apegaron a una dieta mediterránea presentaron una prevalencia de $3.7 \%$ de síndrome metabólico, mientras que aquellos con una dieta usual un $6.6 \%$. En otra investigación realizada en Grecia se apoya también el beneficio del apego a una dieta mediterránea para disminuir la prevalencia de estos trastornos metabólicos, señalan que el efecto positivo se relaciona más que todo con un menor consumo de carnes y mayor cantidad de frutas, vegetales $y$ legumbres. Es importante recalcar que el impacto positivo de la dieta mediterránea y hábitos saludables de sueño se ha documentado tanto al utilizarlo en forma preventiva en niños sanos, como en niños que ya padecen el síndrome metabólico $(8,22)$.

Manejar el sobrepeso y obesidad en niños es complicado, no hay un tratamiento único, sino que requiere combinación de estrategias psicosociales, en estilo de vida e incluso farmacológicas, en algunos casos. Se debe recalcar que abordar este padecimiento desde edades tempranas presenta la ventaja de que incluso una pérdida de peso relativamente pequeña puede mejorar importantemente la salud física del paciente. Una de las propuestas que ha demostrado mayor efectividad de tratamiento, es incidir en el estilo de vida mediante la terapia conductual y cognitivo conductual para modifica conductas, de todas la más exitosa ha sido la que se basa en el apoyo familiar y que además promueve una alta autoeficiencia. Dicha estrategia es segura y se centra en involucrar a la familia como soporte para el paciente en los cambios que va a realizar y el mantenimiento de estos en el tiempo, y a su vez la autoeficiencia en el control de emociones; el 
objetivo es lograr una dieta más saludable, aumento de la actividad física y reducción del sendentarismo (1).

Cada alteración metabólica presente en los pacientes requerirá de un abordaje específico, con intervenciones médica, farmacológicas y otras disciplinas, sin embargo, esto se encuentra fuera de los criterios de inclusión de esta revisión.

\section{CONCLUSIONES}

No existe aún en pediatría consenso en cuanto a niveles serológicos y antropométricos para el diagnóstico de este síndrome, y estos parámetros pueden variar según la raza y edad del individuo. De acuerdo con la evidencia analizada, el estilo de vida de un individuo, en cuanto a alimentación, hábitos de sueño, actividad física, exposición a contaminación ambiental, entre otros... impacta de forma positiva o negativa, según sea el caso, desde la vida intrauterina, llegando a trascender hasta la edad adulta. Existen factores no modificables, como la genética, y otros muchos que sí son modificables, por lo que el esfuerzo del personal de salud debe orientarse a la prevención y abordaje estas alteraciones metabólicas, teniendo en cuenta que con la intervención a menor edad y más pronto en el curso de las alteraciones metabólica, se obtiene mayor impacto positivo, sobre todo en lo que a reducción de peso respecta. Preventivamente se pueden promover la lactancia materna, estilos de vida activos, menor tiempo de uso de pantallas, dieta saludable, tiempo de sueño adecuado para la edad, con inicio de sueño antes de las 22:00 horas debido al ciclo circadiano de las hormonas que regulan el metabolismo. Debido a los múltiples factores que llevan al padecimiento de estos trastornos metabólicos, se sugiere un abordaje multidisciplinario, que contemple factores psicológicos, sociales, conductuales y médicos, se sugiere como posible primera línea la terapia cognitivo conductual para modificación de hábitos y su mantenimiento en el tiempo. Aún faltan más estudios sobre este padecimiento en el campo de la pediatría para generar recomendaciones más estandarizadas para esta población.

\section{Los autores declaran no tener conflicto de interés.}

\section{REFERENCIAS}

1. Miri SF, Javadi M, Lin C-Y, Griffiths MD, Björk M, Pakpour AH. Effectiveness of cognitive-behavioral therapy on nutrition improvement and weight of overweight and obese adolescents: A randomized controlled trial. Diabetes Metab Syndr. 2019;13(3):2190-7.

2. García AG, Urbina Treviño MV, Villalpando Sánchez DC, Aguilar CA. Diagnostic accuracy of triglyceride/glucose and triglyceride/HDL index as predictors for insulin resistance in children with and without obesity. Diabetes Metab Syndr. 2019;13(4):2329-34.

3. Hirschler V, Oestreicher K, Maccallini G, Aranda C. Relationship between obesity and metabolic syndrome among Argentinean elementary school children. Clin Biochem. 2010;43(4-5):435-41.

4. Wong-McClure RA, Gregg EW, Barceló A, Lee K, Abarca-Gómez L, Sanabria-López L, et al. Prevalence of metabolic syndrome in Central America: a cross-sectional population-based study. Rev Panam Salud Publica. 2015;38(3):202-8.

5. Peña-Espinoza BI, Granados-Silvestre M de LÁ, Sánchez-Pozos K, Ortiz-López MG, Menjivar M. Metabolic syndrome in Mexican children: Low effectiveness of diagnostic definitions. Endocrinol Diabetes Nutr (Engl ). 2017;64(7):369-76.

6. Ahrens $W$, Moreno LA, Mårild $S$, Molnár $D$, Siani $A$, De Henauw $S$, et al. Metabolic syndrome in young children: definitions and results of the IDEFICS study. Int J Obes (Lond). 2014;38 Suppl 2(S2):S4-14.

7. Zhang J-S, Gui Z-H, Zou Z-Y, Yang B-Y, Ma J, Jing $\mathrm{J}$, et al. Long-term exposure to ambient air pollution and metabolic syndrome in children and adolescents: A national cross-sectional study in China. Environ Int. 2021;148(106383):106383.

8. Katsa ME, loannidis A, Zyga S, Tsironi M, Koutsovitis $\mathrm{P}$, Chatzipanagiotou $\mathrm{S}$, et al. The effect of nutrition and sleep habits on predisposition for Metabolic Syndrome in Greek children. J Pediatr Nurs. 2018;40:e2-8. 
9. Formisano A, Bammann $\mathrm{K}$, Fraterman A, Hadjigeorgiou C, Herrmann D, lacoviello $L$, et al. Efficacy of neck circumference to identify metabolic syndrome in 3-10 year-old European children: Results from IDEFICS study. Nutr Metab Cardiovasc Dis. 2016;26(6):510-6.

10. Duan $Y$, Sun J, Wang M, Zhao M, Magnussen CG, $\mathrm{Xi}$ B. Association between short sleep duration and metabolic syndrome in Chinese children and adolescents. Sleep Med. 2020;74:343-8.

11. Iguacel I, Michels N, Ahrens W, Bammann K, Eiben G, Fernández-Alvira JM, et al. Prospective associations between socioeconomically disadvantaged groups and metabolic syndrome risk in European children. Results from the IDEFICS study. Int J Cardiol. 2018;272:333-40.

12. Orr CJ, Ben-Davies M, Ravanbakht SN, Yin HS, Sanders LM, Rothman RL, et al. Parental feeding beliefs and practices and household food insecurity in infancy. Acad Pediatr. 2019;19(1):809.

13. Bélanger $M$, Dugas $C$, Perron J, Ruchat S-M, Weisnagel SJ, Marc I, et al. Association between lifestyle habits and adiposity values among children exposed and unexposed to gestational diabetes mellitus in utero. Diabetes Metab Syndr. 2019;13(5):2947-52.

14. Wood CT, Witt WP, Skinner AC, Yin HS, Rothman $\mathrm{RL}$, Sanders LM, et al. Effects of breastfeeding, formula feeding, and complementary feeding on rapid weight gain in the first year of life. Acad Pediatr. 2021;21(2):288-96.

15. Fazeli M, Mohammad-Zadeh M, Darroudi S, Meshkat Z, Moslem A, Ghazizadeh H, et al. New anthropometric indices in the definition of metabolic syndrome in pediatrics. Diabetes Metab Syndr. 2019;13(3):1779-84.

16. Flynn JT, Kaelber DC, Baker-Smith CM, et al. Clinical Practice Guideline for Screening and Management of High Blood Pressure in Children and Adolescents. Pediatrics. 2017;140(3):e20171904.

17. Alliu M K-L, Bertoglia A MP. Evaluación del screening de perfil lipídico propuesto por la Academia Americana de Pediatría. Rev Chil Pediatr. 2012;83(3):231-8.

18. Ritchie SK, Murphy EC-S, Ice C, Cottrell LA, Minor $\mathrm{V}$, Elliott $\mathrm{E}$, et al. Universal versus targeted blood cholesterol screening among youth: The CARDIAC project. Pediatrics. 2010;126(2):260-5.

19. AAP COMMITTEE ON PRACTICE AND AMBULATORY MEDICINE and AAP BRIGHT FUTURES PERIODICITY SCHEDULE WORKGROUP. 2017 Recommendations for Preventive Pediatric Health Care. Pediatrics. 2017;139(4):e20170254.
20. McCormick DP, Reyna L, Reifsnider E. Calories, caffeine and the onset of obesity in young children. Acad Pediatr. 2020;20(6):801-8.

21. Reifsnider E, McCormick DP, Cullen KW, Todd M, Moramarco MW, Gallagher MR, et al. Randomized controlled trial to prevent infant overweight in a high-risk population. Acad Pediatr. 2018;18(3):324-33.

22. Martino F, Puddu PE, Lamacchia F, Colantoni C, Zanoni C, Barillà F, et al. Mediterranean diet and physical activity impact on metabolic syndrome among children and adolescents from Southern Italy: Contribution from the Calabrian Sierras Community Study (CSCS). Int $\mathrm{J}$ Cardiol. 2016;225:284-8. 